\title{
An Explicit Jordan Decomposition of Companion Matrices $^{1}$
}

F.S.V. BAZÁN², Departamento de Matemática, CFM, UFSC, 88040-900, Florianópolis, $\mathrm{SC}$, Brazil.

S. GRATTON 3 CERFACS, 42 Av. Gaspard Coriolis, Toulouse Cedex 01 France.

\begin{abstract}
We derive a closed form for the Jordan decomposition of companion matrices including properties of generalized eigenvectors. As a consequence, we provide a formula for the inverse of confluent Vandermonde matrices and results on sensitivity of multiple roots of polynomials.
\end{abstract}

\section{Introduction}

We are concerned with the Jordan form of companion matrices of the form

$$
C=\left[e_{2}, e_{3}, \ldots, e_{m-1},-a\right],
$$

where $e_{i}$ denotes the $i$ th column of $I$, the $m \times m$ identity matrix, and $a^{T}=$ $\left[a_{0}, a_{1}, \ldots, a_{m-1}\right]$ with $a_{i} \in \mathbb{C}$ (superscript $T$ denotes the transpose of a vector or matrix). Such a form plays an essential role in solving a variety of problems in science and engineering $[1,2,3,5,9,11]$. Let $\lambda_{1}, \ldots, \lambda_{p}$ denote the $p$ distinct eigenvalues of $C$ and let $m_{1}, \ldots, m_{p}$ denote their respective algebraic multiplicities. Then, since $C$ is a nonderogatory matrix $[8,10]$, a particular Jordan decomposition of $C$ can be written as

$$
\left(\begin{array}{ccc}
J_{\lambda_{1}} & & \\
& \ddots & \\
& & J_{\lambda_{p}}
\end{array}\right)=\left[\begin{array}{c}
L_{1} \\
\vdots \\
L_{p}
\end{array}\right] C\left[R_{1} \ldots R_{p}\right] \equiv L C R,
$$

where for $i=1, \ldots p$,

$$
J_{\lambda_{i}}=\left(\begin{array}{cccc}
\lambda_{i} & 1 & & \\
& \ddots & \ddots & \\
& & \lambda_{i} & 1 \\
& & & \lambda_{i}
\end{array}\right) \in \mathbb{C}^{m_{i} \times m_{i}}
$$

and

$$
L R=R L=I,
$$

\footnotetext{
${ }^{1}$ This work was supported partially by CNP, grant 302486/2004-6.

${ }^{2}$ fermin@mtm.ufsc.br

3 gratton@cerfacs.fr
} 
where $R_{i} \in \mathbb{C}^{m \times m_{i}}$ and $L_{i} \in \mathbb{C}^{m_{i} \times m}$. The columns of $R_{i}$ (resp. $L_{i}^{*}$ ) represent a right (resp. left ) Jordan chain associated with $\lambda_{i}$ the leading eigenvector being $R_{i} e_{1}^{\left[m_{i}\right]}$ (resp. $L^{*} e_{m_{i}}^{\left[m_{i}\right]}$ ). The star symbol denotes conjugate transpose, i.e., $L^{*}=\bar{L}^{T}$, and $e_{j}^{\left[m_{i}\right]}$ is the $j$-th column of the $m_{i} \times m_{i}$ identity matrix.

Companion matrix $C$ has the property that the coefficients of its characteristic polynomial are the the $a$ 's themselves: $\pi(t)=\operatorname{det}(t I-C)=a_{0}+a_{1} t+\cdots+$ $a_{m-1} t^{m-1}+t^{m}$. Because of this, issues regarding roots of polynomials can be discussed using the Jordan decomposition of $C$. The goal of this work is to describe a closed form for the Jordan decomposition of $C$, concentrating on properties of generalized eigenvectors and issues regarding the sensitivity of the roots of $\pi$.

\section{Explicit Jordan Decomposition}

We start by providing a technical result.

Lemma 1. For arbitrary $\lambda \in \mathbb{C}$ we set $\phi(\lambda)=\left[1, \lambda, \ldots, \lambda^{m-1}\right]^{T} \in \mathbb{C}^{m}$ and define by $\phi^{(m)}(\lambda)$ the $m$-th derivative of $\phi(\lambda)$ with respect to $\lambda$. Let $H$ be the $m \times m$ matrix

$$
H=\left[\begin{array}{ccccc}
a_{1} & a_{2} & \cdots & a_{m-1} & 1 \\
a_{2} & \cdots & a_{m-1} & 1 & \\
\vdots & \vdots & 1 & & \\
a_{m-1} & 1 & & & \\
1 & & & &
\end{array}\right]
$$

Then for any integers $i$ and $j$, there holds $\frac{\phi^{(i) T}(\lambda)}{i !} H \frac{\phi^{(j)}(\lambda)}{j !}=\frac{\pi^{(i+j+1)}(\lambda)}{(i+j+1) !}$.

Proof. The proof is done by induction on $i$ without difficulties.

Proposition 2. Define $r_{i}=H \frac{\phi^{(i-1)}\left(\lambda_{l}\right)}{(i-1) !}$. The set of vectors $\left\{r_{1}, \cdots, r_{m_{l}}\right\}$ is a right Jordan chain of $C$ associated with the eigenvalue $\lambda_{l}$ and $r_{1}$ is the leading right eigenvector. Similarly, define $\breve{l}_{i}=\frac{\bar{\phi}^{\left(m_{l}-i\right)}\left(\lambda_{l}\right)}{\left(m_{l}-i\right) !}$. The set $\left\{\breve{l}_{1}, \cdots, \breve{l}_{m_{l}}\right\}$ is a left Jordan chain of $C$ associated with the eigenvalue $\lambda_{l}$ and $\breve{l}_{m_{l}}$ is the leading left eigenvector. The left and right generalized Jordan chains are normalized so that

$$
\breve{L}_{l} R_{l} \equiv\left[\begin{array}{c}
\breve{l_{1}^{*}} \\
\vdots \\
\breve{l_{m_{l}}^{*}}
\end{array}\right]\left[r_{1} \ldots r_{m_{l}}\right]=\left(\begin{array}{ccccc}
\alpha_{1} & \alpha_{2} & \cdot & \alpha_{m_{l-1}} & \alpha_{m_{l}} \\
& \cdot & \cdot & \cdot & \alpha_{m_{l-1}} \\
& & \cdot & \cdot & \cdot \\
& & & \cdot & \alpha_{2} \\
& & & & \alpha_{1}
\end{array}\right) \equiv F_{l},
$$

where $\alpha_{i}=\frac{\pi^{\left(m_{l}+i-1\right)}\left(\lambda_{l}\right)}{\left(m_{l}+i-1\right) !}$.

Proof. For arbitrary $\lambda$ of multiplicity $q$ consider the vectors $r_{1}, \ldots, r_{q}$. It is clear that these vectors are linearly independent. Thus, if we set $r_{0}=0$, we have to prove that $r_{1}$ is a right eigenvector of $C$ associated with $\lambda$ and that

$$
(C-\lambda I) r_{j}=r_{j-1}, \quad 1 \leq j \leq q .
$$


For this, if $x=\left[x_{1} \cdots x_{m}\right]^{T}$ is a right eigenvector of $C$ associated with $\lambda$ then

$$
C x=\lambda x \Longleftrightarrow\left\{\begin{array}{ccc}
-a_{0} x_{m} & = & \lambda x_{1} \\
x_{1}-a_{1} x_{m} & = & \lambda x_{2} \\
\vdots & & \\
x_{m-1}-a_{m-1} x_{m} & = & \lambda x_{m}
\end{array}\right.
$$

This shows that $x_{m}$ cannot vanish otherwise $x$ would be the 0 vector. Setting $x_{m}=1$ it is easy to see that $x=H \phi(\lambda)$ and thus one has

$$
C H \phi(\lambda)=\lambda H \phi(\lambda) \text {. }
$$

We now prove conditions (2.3). Taking derivative with respect to $\lambda$ in (2.4) we have

$$
C H \phi^{(1)}(\lambda)=H \phi(\lambda)+\lambda H \phi^{(1)}(\lambda) .
$$

This shows that (2.3) holds for $j=2$, and an inductive argument obtained by repeated differentiation of (2.5) concludes the proof in the case of the right generalized eigenvectors. A similar proof can be obtained for the generalized left eigenvectors by starting with $\phi(\lambda)^{T} C^{T}=\phi(\lambda)^{T} \lambda$ instead of (2.4) and taking the derivatives of this equality. The normalization factors $\alpha_{i}$ are a consequence of Lemma 1.

To obtain the Jordan decomposition, we transform the left Jordan chain so that the normalization (1.2) holds.

Proposition 3. Define $L_{l}^{*}=\left[l_{1}, \ldots, l_{m_{l}}\right]=\breve{L}_{l}^{*} F_{l}^{-*}$. The set $\left\{l_{1}, \ldots, l_{m_{l}}\right\}$ is a left Jordan chain of $C$ associated with the eigenvalue $\lambda_{l}, l_{m_{l}}$ being the leading left eigenvector. The left and right generalized Jordan chains are normalized so that $L_{l} R_{l}=$ $I \in \mathbb{R}^{m_{l} \times m_{l}}$. Similarly, if $\breve{R}_{l}=\left[\breve{r}_{1}, \ldots, \breve{r}_{m_{l}}\right]=\left[r_{1}, \ldots r_{m_{l}}\right] F_{l}^{-1}$, then $\left\{\breve{r}_{1}, \ldots, \breve{r}_{m_{l}}\right\}$ is a right Jordan chain of $C$ associated with $\lambda_{l}$, and $\breve{L}_{l} \breve{R}_{l}=I \in \mathbb{R}^{m_{l} \times m_{l}}$.

Proof. Let $\gamma_{i}$ be defined by the recursion

$$
\begin{aligned}
& \gamma_{1}=1 / \alpha_{1} \\
& \gamma_{i+1}=-\frac{1}{\alpha_{1}} \sum_{k=1}^{i} \alpha_{i-k+2} \gamma_{k}, \quad i=1, \ldots m_{l}-1
\end{aligned}
$$

in such a way that

$$
G=\left(\begin{array}{ccccc}
\gamma_{1} & \gamma_{2} & \cdot & \gamma_{m_{l-1}} & \gamma_{m_{l}} \\
& \cdot & \cdot & \cdot & \gamma_{m_{l-1}} \\
& & \cdot & \cdot & \cdot \\
& & & \cdot & \gamma_{2} \\
& & & & \gamma_{1}
\end{array}\right)=F_{l}^{-1}
$$

The set $\left\{\overline{\breve{l}}_{m_{l}} \ldots \overline{\breve{l}}_{1}\right\}$ forms a right Jordan chain of $C^{T}$ associated with $\lambda_{l}$. For any nonsingular matrix $X$ commuting with $J_{\lambda_{l}},\left[\overline{\breve{l}}_{m_{l}} \ldots \overline{\breve{l}}_{1}\right] X$ is a right Jordan chain $C^{T}$ associated with $\lambda_{l}$. By definition of the $l_{i}$ 's, $\left[\begin{array}{llll}l_{m_{l}} & \ldots & l_{1}\end{array}\right]=\left[\begin{array}{llll}\breve{l}_{m_{l}} & \ldots & \breve{l}_{1}\end{array}\right] \bar{G}$. A direct computation shows that $G$ commutes with $J_{\lambda_{l}}$, which implies that $\left\{\bar{l}_{m_{l}}, \cdots, \bar{l}_{1}\right\}$ is a right Jordan chain of $C^{T}$ associated with $\lambda_{l}$, that is, $\left\{l_{l}, \ldots, l_{m_{l}}\right\}$ 
is a left Jordan chain of $C$ associated with $\lambda_{l}$. Additionally, since by definition of the $\gamma_{i}$ 's, $G F_{l}=F_{l} G=I$, it follows

$$
\left[\begin{array}{c}
l_{1}^{*} \\
\vdots \\
l_{m_{l}}^{*}
\end{array}\right]\left[r_{1} \ldots r_{m_{l}}\right]=G\left[\begin{array}{c}
\breve{l_{1}^{*}} \\
\vdots \\
\breve{l_{m_{l}}^{*}}
\end{array}\right]\left[r_{1} \ldots r_{m_{l}}\right]=I,
$$

and the first part of the proposition is proved. The proof of the remaining part is a consequence of Eq. (2.2) since $\left[r_{1} \ldots r_{m_{l}}\right] F_{l}^{-1}$ is a right Jordan chain of $C$ associated with $\lambda_{l}$ as we have seen that $F^{-1}$ commutes with $J_{\lambda_{l}}$.

An immediate consequence of Prop. 3 is an explicit formula for computing the inverse of confluent Vandermonde matrices as described below

Corollary 4 (Inversion formula). Let $\breve{L}$ be the confluent Vandermonde matrix defined by $\breve{L}^{*}=\left[\breve{L}_{1}^{*} \ldots \breve{L}_{p}^{*}\right]$. Then

$$
\breve{L}^{-1}=\left[R_{1} \cdots R_{p}\right] F^{-1} \quad \text { with } \quad F=\operatorname{diag}\left(F_{1}, \ldots, F_{p}\right) .
$$

\subsection{Numerical illustration: Jordan decomposition}

We present an illustration of the above notions for $m=5,\left(\lambda_{1}, m_{1}\right)=(1,2)$, $\left(\lambda_{2}, m_{2}\right)=(2,2),\left(\lambda_{3}, m_{3}\right)=(3,1)$, in which case, $\pi(t)=(t-1)^{2}(t-2)^{2}(t-3)$. We show how to obtain easily a Jordan form of the companion matrix associated with $\pi$. Note that $\pi(t)=t^{5}-9 t^{4}+31 t^{3}-51 t^{2}+40 t-12$.

- Case of $\lambda=1, m_{1}=2$.

From $(t-2)^{2}(t-3)=t^{3}-7 t^{2}+16 t-12$ and $(t-1)(t-2)^{2}(t-3)=$ $t^{4}-8 t^{3}+23 t^{2}-28 t+12$ follows using Prop. 5 and the definition of $\breve{l}_{i}$ that

$$
R_{1}=\left(\begin{array}{rr}
12 & -12 \\
-28 & 16 \\
23 & -7 \\
-8 & 1 \\
1 & 0
\end{array}\right) \text { and } \breve{L}_{1}^{*}=\left(\begin{array}{ll}
0 & 1 \\
1 & 1 \\
2 & 1 \\
3 & 1 \\
4 & 1
\end{array}\right)
$$

From $\pi^{(2)}(1) / 2=-2$ and $\pi^{(3)}(1) / 6=5$, we obtain

$$
F_{1}=\left(\begin{array}{rr}
-2 & 5 \\
0 & -2
\end{array}\right), F_{1}^{-1}=\frac{1}{4}\left(\begin{array}{rr}
-2 & -5 \\
0 & -2
\end{array}\right) \text {, and } L_{1}^{*}=\breve{L}_{1}^{*} F_{1}^{-*}=\frac{1}{4}\left(\begin{array}{rr}
-5 & -2 \\
-7 & -2 \\
-9 & -2 \\
-11 & -2 \\
-13 & -2
\end{array}\right) \text {. }
$$

- The same calculation for the two remaining roots gives

$$
R=\left[R_{1}, R_{2}, R_{3}\right]=\left(\begin{array}{rrrrr}
12 & -12 & 6 & -3 & 4 \\
-28 & 16 & -17 & 7 & -12 \\
23 & -7 & 17 & -5 & 13 \\
-8 & 1 & -7 & 1 & -6 \\
1 & 0 & 1 & 0 & 1
\end{array}\right)
$$


and

$$
L^{*}=\left[L_{1}^{*}, L_{2}^{*}, L_{3}^{*}\right]=\frac{1}{4}\left(\begin{array}{rrrrr}
-5 & -2 & 4 & -4 & 1 \\
-7 & -2 & 4 & -8 & 3 \\
-9 & -2 & 0 & -16 & 9 \\
-11 & -2 & -16 & -32 & 27 \\
-13 & -2 & -64 & -64 & 81
\end{array}\right),
$$

yielding a Jordan decomposition $R J L=C$, where $J$ (a Jordan matrix) and $C$ are of the form

$$
J=\left(\begin{array}{lllll}
1 & 1 & 0 & 0 & 0 \\
0 & 1 & 0 & 0 & 0 \\
0 & 0 & 2 & 1 & 0 \\
0 & 0 & 0 & 2 & 0 \\
0 & 0 & 0 & 0 & 3
\end{array}\right), \quad C=\left(\begin{array}{rrrrr}
0 & 0 & 0 & 0 & 12 \\
1 & 0 & 0 & 0 & -40 \\
0 & 1 & 0 & 0 & 51 \\
0 & 0 & 1 & 0 & -31 \\
0 & 0 & 0 & 1 & 9
\end{array}\right) .
$$

\section{Generalized Eigenvector Properties}

It is known that right eigenvectors of companion matrices like $C$ in (1.1) can be computed by finding the vector of coefficients of $\pi(t) /\left(t-\lambda_{l}\right)$ (see, e.g., Toh and Trefethen [6] or Bezerra and Bazán [3, Prop. 2.4]). In our notation this reads

$$
\pi_{1}(t)=\pi(t) /\left(t-\lambda_{l}\right) \equiv \phi(t)^{T} r_{1} .
$$

In what follows we extend this result to the complete right Jordan chain.

Proposition 5. Define $\pi_{i}(t)=\phi(t)^{T} r_{i}\left(i=1, \ldots, m_{l}\right)$ where $r_{i}$ are generalized right eigenvectors of $C$ as introduced in Prop. 2. Then $\pi_{i}$ is a monic polynomial of degree $m-i$ of the form

$$
\pi_{i}(t)=\left(t-\lambda_{l}\right)^{m_{l}-i} \prod_{\substack{j=1 \\ j \neq l}}^{p}\left(t-\lambda_{j}\right)^{m_{j}} .
$$

Proof. It is clear that all $\pi_{i}$ are monic polynomials of degree $m-i$. The definition of the $r_{i}$ 's and successive differentiation imply

$$
\begin{array}{ccc}
\pi_{1}(t) & = & \phi^{T}(t) H \phi\left(\lambda_{l}\right) \\
\pi_{1}^{(1)}(t) & = & \phi^{(1)^{T}}(t) H \phi\left(\lambda_{l}\right) \\
\vdots & & \\
\pi_{1}^{(i)}(t) & = & \phi^{(i)^{T}}(t) H \phi\left(\lambda_{l}\right) \\
\vdots & & \\
\pi_{1}^{\left(m_{l}-1\right)}(t) & = & \phi^{\left(m_{l}-1\right)^{T}}(t) H \phi\left(\lambda_{l}\right) .
\end{array}
$$

If $t=\lambda_{l}$, Prop. 1 implies that for $i=1, \ldots, m_{l},(3.2)$ becomes

$$
\pi_{i}\left(\lambda_{l}\right)=\pi_{1}^{(i-1)}\left(\lambda_{l}\right)=(i-1) ! \phi^{T}\left(\lambda_{l}\right) H \frac{\phi^{(i-1)}\left(\lambda_{l}\right)}{(i-1) !}=(i-1) ! \pi^{i-1}\left(\lambda_{l}\right) .
$$


But since $\lambda_{l}$ is a multiple root of $\pi$, this equality implies that $\lambda_{l}$ is a root of $\pi_{i}$ $\left(i=1 \ldots m_{l}-1\right)$ and a recursive argument shows that this root is of multiplicity $m_{l}-i$. If $t=\lambda_{k} \neq \lambda_{l}$, a similar procedure and the existing biorthogonality condition between left and right generalized eigenvectors leads to

$$
\pi_{i}\left(\lambda_{k}\right)=\pi_{1}^{(i-1)}\left(\lambda_{k}\right)=(i-1) ! \phi^{T}\left(\lambda_{l}\right) H \frac{\phi^{(i-1)}\left(\lambda_{k}\right)}{(i-1) !}=0,
$$

which concludes the proof.

It is instructive to describe the meaning of this proposition. Let $C_{i}(i=$ $\left.1, \ldots, m_{l}-1\right)$ denote the $(m-i) \times(m-i)$ companion matrix associated with the polynomial $\pi_{i}$ and, for $i=1, \ldots, m_{l}$, let $\check{r}_{i}$ be the vector formed by taking the first $m-i+1$ components of $r_{i}$. Then, with the convention that $C_{0}=C$, the proposition ensures that

$$
C_{i-1} \check{r}_{i}=\lambda_{l} \check{r}_{i}, \quad i=1, \ldots m_{l},
$$

and $\lambda_{l}$ is a simple eigenvalue of the companion matrix $C_{m_{l}-1}$. For future reference, the left eigenvector of of $C_{m_{l}-1}$, will be denoted by $\psi\left(\lambda_{l}\right)$. It is defined by

$$
\psi\left(\lambda_{l}\right)=\left[1, \bar{\lambda}_{l}, \cdots, \bar{\lambda}_{l}^{m-m_{l}}\right]^{T} .
$$

\section{Condition Estimation}

We analyze the sensitivity of the roots of $\pi(t)$ to perturbations in the coefficients $a_{j}$ viewing the roots as eigenvalues of the companion matrix $C$. Let $\widetilde{\pi}(t)$ denote the monic polynomial with coefficients $\widetilde{a}_{i}=a_{i}+\Delta a_{i}$ and let $\widetilde{C}$ denote the associated companion matrix. Then, depending on the way the perturbations $\widetilde{a}_{j}$ are measured, different condition numbers for $\lambda$ can be obtained. Suppose for instance that the $\Delta a_{j}$ 's are assumed to satisfy the componentwise inequalities

$$
\left|\Delta a_{i}\right| \leq \epsilon \alpha_{i}, i=0, \ldots, m-1,
$$

where $\alpha_{j}$ are arbitrary non negative real numbers, and let $\widetilde{\lambda}_{j}, j=1, \ldots d$, denote the eigenvalues of $\widetilde{C}$ for $\epsilon$ small enough. For the so-called componentwise model of perturbations defined by (4.1), we have the definition below, where for simplicity, $\lambda_{l}$ and its corresponding multiplicity $m_{l}$ will be denoted by $\lambda$ and $d$, respectively, and $|\Delta \lambda|=\max _{j=1, \ldots, d}\left|\lambda-\widetilde{\lambda}_{j}\right|$.

Definition 6. [4] The componentwise relative condition number of the root $\lambda$ of multiplicity $d$ is defined by

$$
\kappa^{\mathcal{C}}(\lambda)=\lim _{\epsilon \rightarrow 0} \sup _{\left|\Delta a_{j}\right| \leq \epsilon \alpha_{j}} \frac{|\Delta \lambda|}{|\lambda| \epsilon^{1 / d}} .
$$

A precise description of $\kappa^{\mathcal{C}}(\lambda)$ is given in the proposition below. 
Proposition 7. Suppose the perturbations $\Delta a_{j}$ satisfy (4.1). Then the componentwise relative condition number of the root $\lambda$ of multiplicity $d, \kappa^{\mathcal{C}}(\lambda)$, is

$$
\kappa^{\mathcal{C}}(\lambda)=\frac{1}{|\lambda|}\left(\frac{d ! \sum_{j=0}^{m-1}\left|\lambda^{j}\right| \alpha_{j}}{\left|\pi^{(d)}(\lambda)\right|}\right)^{1 / d} .
$$

Proof. A sketch of the proof is as follows. Let $\{\widetilde{\lambda}, \widetilde{r}\}$ be a right eigenpair of $\widetilde{C}=$ $C+\Delta C$. If $\widetilde{\lambda}=\lambda+\Delta \lambda, \widetilde{r}=r+\Delta r$, then $(C+\Delta C)(r+\Delta r)=(\lambda+\Delta \lambda)(r+\Delta r)$, iff $C \Delta r+\Delta C r+\Delta C \Delta r=\lambda \Delta r+\Delta \lambda r+\Delta \lambda \Delta r$. Now since by by Prop. 2.2, the right eigenvector $\widetilde{r}$ of $\widetilde{C}$ satisfies $r+\Delta r=\widetilde{H} \phi(\widetilde{\lambda})$, where $\widetilde{H}=H+\Delta H$ has the same structure as $H$ but with entries $\widetilde{a}_{j}=a_{j}+\Delta a_{j}$, it can be proved that the $m$-th component of $\Delta r$ equals zero. From this and the fact that $\Delta C=-\Delta a e_{m}^{[m] *}$ where $\Delta a=\left[\Delta a_{0}, \ldots, \Delta a_{m-1}\right]^{T}$ gives $\Delta C \Delta r=0$. Some algebraic manipulations lead then to the following first order result

$$
\Delta \lambda^{d}=\frac{d ! \phi^{T}(\lambda) \Delta a}{\pi^{(d)}(\lambda)}
$$

from which the proof follows.

Remark. Another condition number for $\lambda$ can be readily obtained when the perturbations are assumed to satisfy

$$
\|\Delta a\|_{2} \leq \delta \alpha,
$$

where $\alpha$ is an arbitrary positive real number (e.g., $\alpha=\|a\|_{2}$ ). This gives rise to the so-called normwise relative condition number $\kappa(\lambda)$ which is given by

$$
\kappa(\lambda)=\frac{1}{|\lambda|}\left(\frac{d !\|\phi(\lambda)\|\|a\|}{\left|\pi^{(d)}(\lambda)\right|}\right)^{1 / d} .
$$

When the perturbations are measured in a normwise absolute sense, i.e., when $\alpha=1$ in (4.5), the normwise absolute condition $\kappa_{a}(\lambda)$ can be shown to satisfy

$$
\kappa_{a}(\lambda)=|\lambda| \kappa(\lambda)
$$

Observe that the multiple eigenvalue $\lambda$ can be viewed as a simple eigenvalue of the companion matrix $C_{d-1}$ in (3.3) (see the remark after Prop. 5). In what follows we shall see that $\kappa_{a}(\lambda)$ and $\kappa_{a}(\lambda)$ can be related to the Wilkinson condition number of $\lambda$ viewed as a simple eigenvalue of $C_{d-1}$; this condition number is defined by [12]

$$
\kappa_{\mathrm{W}}(\lambda)=\frac{\|\psi(\lambda)\|_{2}\left\|\check{r}_{d}\right\|_{2}}{\left|\psi^{*}(\lambda) \check{r}_{d}\right|} .
$$

Proposition 8. Assume that the perturbation $\Delta a$ satisfies the model (4.5) with $\alpha=\|a\|_{2}$. Then the condition number $\kappa(\lambda)$ given in (4.6) satisfies

$$
\kappa(\lambda) \leq \frac{1+|\lambda|}{|\lambda|}\left(\frac{\|a\|_{2}^{2}}{1+\|a\|_{2}^{2}}\right)^{\frac{1}{2 d}} \kappa_{W}(\lambda)^{1 / d}\left(\frac{\|\phi(\lambda)\|_{2}}{\|\psi(\lambda)\|_{2}}\right)^{1 / d} .
$$


Proof. Observe that using Prop. 2 and the property $\phi^{T}(\lambda) r_{d}=\psi^{*}(\lambda) \check{r}_{d}$ (see (3.3) again), (4.6) can be rewritten as

$$
\kappa(\lambda)=\frac{1}{|\lambda|}\left(\frac{\|\phi(\lambda)\|_{2}\left\|r_{d}\right\|_{2}}{\left|\phi^{T}(\lambda) r_{d}\right|}\right)^{1 / d}\left(\frac{\|a\|_{2}}{\left\|r_{d}\right\|_{2}}\right)^{1 / d}=\frac{1}{|\lambda|} \kappa_{\mathrm{W}}(\lambda)^{1 / d} \frac{\|\phi(\lambda)\|_{2}^{1 / d}}{\|\psi(\lambda)\|_{2}^{1 / d}} \frac{\|a\|_{2}^{1 / d}}{\left\|r_{d}\right\|_{2}^{1 / d}} .
$$

We now recall that vector $r_{d}$ in (4.9) contains the coefficients of the monic polynomial $\pi_{d}$ that is of degree $m-d$ (see the remark after Prop. 5). Set $q=m-d-1$, $r_{d}=\left[c_{0}, c_{1}, \cdots, c_{q}, 1,0 \cdots 0\right]^{T} \equiv\left[\check{r}_{d}^{T}, 0 \cdots 0\right]^{T}$ and collect the coefficients of the polynomial $(t-\lambda)^{d}=b_{0}+b_{1} t+\cdots+b_{d-1} t^{d-1}+t^{d}$ in a vector $b=\left[b_{0}, b_{1}, \cdots, b_{d-1} 1\right]^{T}$. Some algebraic manipulations and Prop. 5 give then

$$
\pi(t)=(t-\lambda)^{d} \pi_{d}(t) \Leftrightarrow\left[\begin{array}{c}
a \\
1
\end{array}\right]=B \check{r}_{d} .
$$

where $B$ is an $(m+1) \times(m-d+1)$ lower triangular Toeplitz matrix whose first column vector is $\left[b_{0}, b_{1}, \ldots, b_{d-1}, 1,0, \ldots, 0\right]^{T}$. Since $\|B\|_{2} \leq \sqrt{\|B\|_{1}\|B\|_{\infty}}=\|B\|_{1}$, (4.10) gives

$$
1+\|a\|_{2}^{2} \leq\|B\|_{2}^{2}\left\|r_{d}\right\|_{2}^{2}=\|B\|_{1}^{2}\left\|r_{d}\right\|_{2}^{2} .
$$

Hence, taking into account that $\|B\|_{1}=(1+|\lambda|)^{d}$, as the $b_{i}$ 's are of the form $b_{i}=\lambda^{d-i} \mathcal{C}_{i}^{d}(i=0, \ldots, d)$ where $\mathcal{C}_{i}^{d}$ are binomial coefficients of the expansion $(t-\lambda)^{d}$, we have $\left\|r_{d}\right\|_{2} \geq \sqrt{1+\|a\|_{2}^{2}} /(1+|\lambda|)^{d}$. The proof follows on replacing this lower bound in (4.9).

An immediate conclusion is that if $d$ is not large and $|\lambda|$ is a moderate number then $\kappa(\lambda)$ essentially depends on $\kappa_{\mathrm{w}}(\lambda)$. Thus if $\lambda$ is a well conditioned eigenvalue of the deflated companion matrix $C_{d-1}$ (or equivalently, a well conditioned simple root of $\left.\pi_{d-1}(t)\right)$ and the ratio $\|\phi(\lambda)\|_{2} /\|\psi(\lambda)\|_{2}$ is rather small, then moderate values for $\kappa(\lambda)$ may be expected. We notice however that even if $\kappa(\lambda)$ is small, the error in $\lambda$ strongly depends on the multiplicity $d$ and the size of the perturbations $\Delta a_{j}$. In general, if the perturbations $\Delta a_{j}$ are small enough, the relative error in $\lambda$ can be estimated by the rule

$$
|\Delta \lambda| /|\lambda| \approx \kappa(\lambda) \delta^{1 / d}
$$

\subsection{Numerical illustration: Condition estimation}

We consider the polynomial $\pi(t)=(t-\lambda)^{5}\left(1+t+\cdots+t^{15}\right)$ with $\lambda=(1+9 s)+s i$, $0 \leq s \leq 2$. This example is designed to illustrate the role of the deflated polynomial $\pi_{d-1}$ (in this case $d=5$, see Prop. 5) in estimating the sensitivity of a multiple root. In fact, as in in this case the deflated polynomial $\pi_{d-1}(t)=(t-\lambda)\left(1+t+\cdots+t^{15}\right)$ reduces to the polynomial $t^{16}-1$ when $\lambda=1$, all roots of which are known to be extremely well-conditioned [7, Example 4.3], small condition numbers for the multiple root $\lambda$ can be expected provided that $\lambda \approx 1$, the conditioning being more favorable for the (simple) roots of $\pi(t)$ (the roots of $1+t+\cdots+t^{15}$ ). Indeed, if the simple roots of $\pi(t)$ are denoted by $\breve{\lambda}_{k}$, it can be proved that

$$
\kappa_{a}(\lambda)=\frac{\left(1+|\lambda|^{2}+|\lambda|^{4}+\cdots+|\lambda|^{38}\right)^{0.1}}{\prod_{k=1}^{15}\left|\lambda-\breve{\lambda}_{k}\right|^{0.2}}, \quad \text { and } \quad \kappa_{a}\left(\breve{\lambda}_{k}\right)=\frac{\sqrt{5}}{8} \frac{\left|\breve{\lambda}_{k}-1\right|}{\left|\breve{\lambda}_{k}-\lambda\right|^{5}}
$$




\begin{tabular}{c|c|c|c|c|c}
\hline \hline$\lambda$ & $\kappa(\lambda)$ & $\kappa_{a}(\lambda)$ & $\kappa_{\mathrm{W}}$ & $\rho$ & $|\Delta \lambda| /|\lambda|$ \\
\hline \hline $19+2 \imath$ & $1.3169 e+1$ & $1.0480 e+1$ & $3.7970 e+0$ & $1.3322 e+5$ & $1.3169 e-1$ \\
$15+1.5 \imath$ & $1.0724 e+1$ & $8.6469 e+0$ & $3.7443 e+0$ & $5.1642 e+4$ & $1.0724 e-1$ \\
$10+\imath$ & $7.4747 e+0$ & $6.2102 e+0$ & $3.6221 e+0$ & $1.0201 e+4$ & $7.4747 e-2$ \\
$5+0.5 \imath$ & $3.9220 e+0$ & $3.4955 e+0$ & $3.2743 e+0$ & $6.3756 e+2$ & $3.9220 e-2$ \\
$1.45+0.05 \imath$ & $1.5800 e+0$ & $1.1384 e+0$ & $1.7266 e+0$ & $4.4310 e+0$ & $1.5800 e-2$ \\
1 & $1.2693 e+0$ & $7.7495 e-1$ & $1.0000 e+0$ & $1.1180 e+0$ & $1.2693 e-2$ \\
\hline
\end{tabular}

Table 1: Condition numbers, ratio $\rho=\|\phi(\lambda)\|_{2} /\|\psi(\lambda)\|_{2}$ and predicted errors .

Some numerical results displayed in Table 1 corresponding to several $\lambda$ 's confirm the theoretical prediction. The table includes condition numbers, the predicted eigenvalue error described in (4.11), and the ratio $\rho=\|\phi(\lambda)\|_{2} /\|\psi(\lambda)\|_{2}$. Also, and mainly to verify the theoretical prediction of the error, approximate roots obtained from polynomials with coefficients $\widetilde{a}_{j}=a_{j}+\Delta a_{j}$ where $\Delta a_{j}$ are random numbers satisfying a normwise relative error $\delta=10^{-10}$, are displayed in Figure 1. All computations were performed using MATLAB.

The results confirm that moderate values of $\kappa(\lambda)$ do not necessarily imply small eigenvalue errors when the multiplicity is rather large and that reasonably small errors can be expected when both the Wilkinson condition number $\kappa_{\mathrm{W}}(\lambda)$ and the ratio $\rho$ are small. The relative insensitivity of simple roots is also apparent, as predicted.

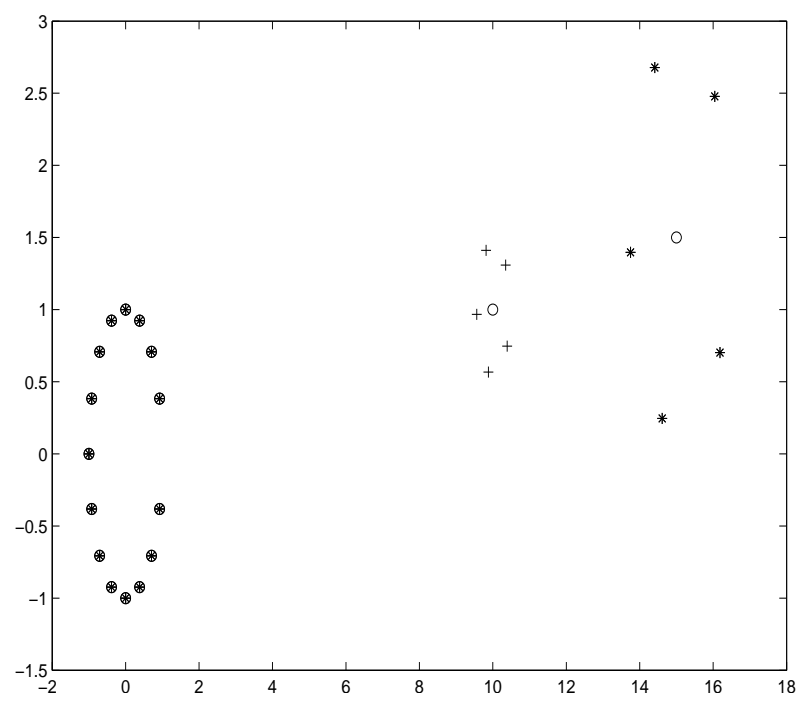

Figure 1: Exact and approximate eigenvalues: Case 1: $\lambda=15+1.5 \imath$. $\circ$ : Exact eigenvalue, * : Approximate eigenvalue. Case 2: $\lambda=10+\imath$. ०: Exact eigenvalue, + : Approximate eigenvalue. 


\section{Final Remark}

We have presented an explicit Jordan decomposition of companion matrices and results on sensitivity of roots of polynomials. Efficient implementation of the formula for the inverse of confluent Vandermonde matrices is the subject of ongoing work. The results on sensitivity of multiple roots, on the other hand, contribute to clarify an important problem in numerical analysis usually overlooked so far.

\section{References}

[1] F.S.V. Bazán, Ph.L. Toint, Error analysis of signal zeros from a related companion matrix eigenvalue problem, Applied Mathematics Letters, 14 (2001), 859-866.

[2] F.S.V. Bazán. Error analysis of signal zeros: a projected companion matrix approach, Linear Algebra Appl., 369 (2003), 153-167.

[3] L.H. Bezerra, F.S.V. Bazán, Eigenvalue locations of generalized predictor companion matrices, SIAM J. Matrix Anal. Appl. 19, No. 4 (1998), 886-897.

[4] F. Chaitin-Chatelin, V. Frayssé, "Lectures on Finite Precision Computations". SIAM, Philadelphia 1996.

[5] M.I. Friswell, U. Prells, S.D. Garvey, Low-rank damping modifications and defective systems, Journal of Sound and Vibration, 279 (2005), 757-774.

[6] K-C Toh, Lloyd N. Trefethen, Pseudozeros of polynomials and pseudospectra of companion matrices, Numer. Math. 68 (1994), 403-425.

[7] W. Gautschi, Questions of numerical condition related to polynomials, in MAAA Studies in Mathematics, Vol. 24, Studies in Numerical Analysis, G. H. Golub, ed., USA, 1984, The Mathematical Association of America, pp. 140177.

[8] G.H. Golub, C.F. Van Loan, "Matrix Computations", The Johns Hopkins University Press, Baltimore, 1996.

[9] P. de Groen, B. de Moor, The fit of a sum of exponentials to noisy data, Comput. Appl. Math., 20 (1987), 175-187.

[10] R. Horn, Ch.R. Johnson, "Matrix Analysis", Cambridge University Press 1999.

[11] H.M. Möller, J. Stetter, Multivariate polynomial equations with multiple zeros solved by matrix eigenproblems, Numer. Math., 70, 311-329.

[12] J.H. Wilkinson, "The Algebraic Eigenvalue Problem", Oxford University Press, Oxford, UK, 1965. 